\title{
A Paper on Detoxification Procedures in North Central Nigeria. Case Study: Federal College of Complementary and Alternative Medicine, Clinic, Abuja, Nigeria
}

ISSN: 2640-9208

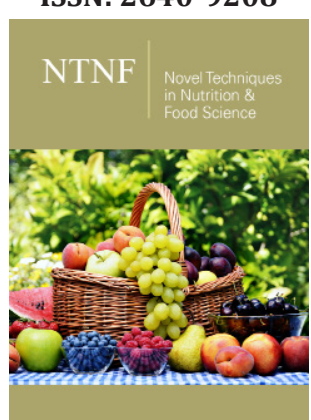

${ }^{* 1}$ Corresponding author: Osunderu OA, Department of Research and Development, Nigeria

Submission: 海 September 16, 2017

Published: 战April 12, 2019

Volume 3 - Issue 5

How to cite this article: Osunderu 0, Jacob E. A Paper on Detoxification Procedures in North Central Nigeria. Case Study: Federal College of Complementary and Alternative Medicine, Clinic, Abuja, Nigeria. Nov Tech Nutri Food Sci. 3(5).NTNF.000571.2019. DOI: $10.31031 / N T N F .2019 .03 .000571$

Copyright@ Osunderu OA, This article is distributed under the terms of the Creative Commons Attribution 4.0 International License, which permits unrestricted use and redistribution provided that the original author and source are credited.
Osunderu OA* and Jacob E

Department of Research \& Development, Nigeria

\begin{abstract}
Over three-quarter of the world's population is using Complementary and Alternative Medicine (CAM) with an increasing trend globally. CAMs may be beneficial but are not completely harmless. This study aimed to assess the trend in the treatments administered to 961 patients that attended the CAM clinic at the Federal College of Complementary and Alternative medicine Utako, Abuja North Central Nigeria for 10 months. This is a retrospective study involving 961 participants who attended FEDCAM clinic and were treated of various ailments like back pain, arthritis, high blood pressure, STDs, and malaria fever for 10 months. A total of about 1, 580 diseases of different levels were examined, and these are classified according to body systems. Duration of treatment varied from $\leq 4$ weeks to $\geq 25$ weeks. $90 \%$ of patients were treated using naturopathic detoxification therapies primarily for symptoms ranging from gastrointestinal disorders, general cleansing/preventive medicine, autoimmune disease and environmental exposure. 961 patients at one point or the other visited the clinic for medical attention. This figure when compared to the previous year shows a remarkable increase which is a justification of the fact that a lot of people are beginning to appreciate the importance of integrative medicine despite the criticisms the institution has witnessed in the recent times.
\end{abstract}

\section{Introduction}

There is an increase of exposure to persistent bio accumulative toxicants (PBTs) by humans and animals. The manufacture, use, and release of PBTs has been associated with adverse health effects, including endocrine disruption [1] neurological [2] and reproductive effect [3] as well as cancers [4] and cardiovascular diseases [5]. Examples of chemicals associated with diseases and disorders include organochlorine pesticides, phthalates, bisphenol A, and polybrominated diphenyl ethers Globally, people have developed unique indigenous healing traditions adapted and defined by their culture, beliefs and environment, which satisfied the health needs of their communities over centuries [6].

Detoxification is one of the more widely used treatments and concepts in complementary and alternative medicine. Clinical toxicology books define detoxification as conversion of "toxic parent compounds to nontoxic metabolites," CDC 2010. It is based on the principle that illnesses can be caused by the accumulation of toxic substances (toxins) in the body. The concentration of these toxic substances in the body, assessed by biomonitoring, is commonly referred to as the "body burden". These toxicants or chemicals come from various environmental sources, such as soil, air, water, dust, or food CDC 2010. Eliminating existing toxins and avoiding new toxins are essential parts of the healing process. Because in recent times, studies have increasingly shown that the human body burden of several chemicals, at physiologically relevant levels, is a real threat to human health, including fetal and neonatal health [7]. Therefore, detoxification has been adopted as one of the main treatment procedures frequently recommended for our patients in the FEDCAM clinic. Detoxification methods of healing have been used for thousands of years, most times it entails "all reactions, enzymecatalyzed or not, that consume toxic metabolites without producing injury." Barile [8]; Sullivan 
[9]. The United States Centre for Disease Control estimate that over $80 \%$ of all illnesses has environmental and lifestyle causes. Detoxification has also become a prominent treatment as people have become more aware of environmental pollution [10].

\section{Methods}

\section{Research design}

This was a qualitative, retrospective study, information was collected in a descriptive manner over a 10-month period, in order to gain a general understanding of complementary/alternative medical detoxification practices. Data are presented mostly in percentage form (Table 1). Means and standard deviations were computed with microsoft excel (Figure 1).

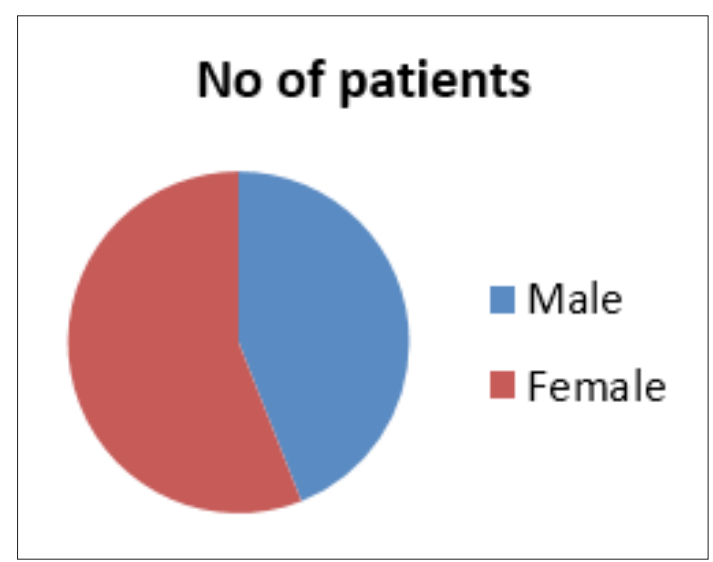

Figure 1: Demographics of Respondents.

Table 1: Demographics of respondents.

\begin{tabular}{|c|c|}
\hline \multicolumn{2}{|c|}{ Total no of Patients: 961} \\
\hline Male patients & 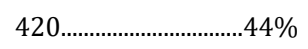 \\
\hline Female patients & $541 \ldots \ldots \ldots$ \\
\hline
\end{tabular}

Results

\section{Treatments administered to patients}

Multiple therapies were utilized for the detoxification procedure: 7 different therapies were used in the detoxification procedure. However, based on the severity of the case being handled, one or more of these therapies could be implored together for treatment. These therapies include; detoxification, massage, homeopathy, acupuncture, pyro energy, hydrotherapy and naturopathy. On the basis of frequency of application of one or more of the therapies above, the following classification was arrived at (Table 2).

Note: It can be seen from the above that Naturopathy alone forms almost half of the treatment (Figure 2). This means that naturopathy was majorly used for detoxification procedure in the patients.

Naturopathy only. . $.40 \%$

Others $.60 \%$

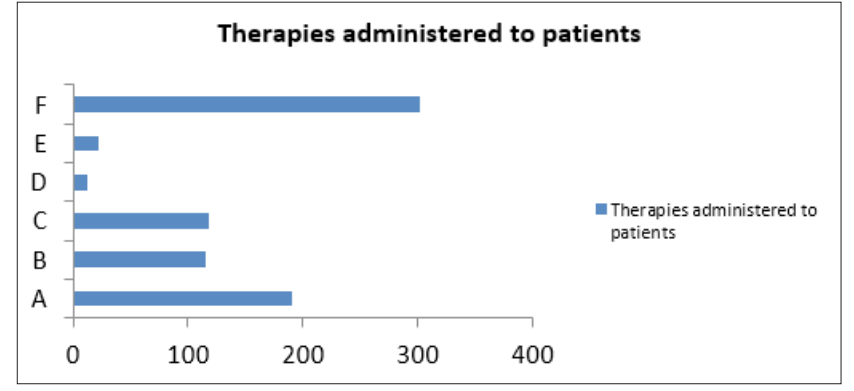

Figure 2:

Table 2:

\begin{tabular}{|c|c|}
\hline Therapies Administered & No of Patients \\
\hline $\begin{array}{c}\text { Detoxification, Pyro energy, Massage, Homeopathy, } \\
\text { Hydrotherapy, Naturopathy }\end{array}$ & 190 \\
\hline $\begin{array}{c}\text { Detoxification, Pyro energy, Massage, Acupuncture, } \\
\text { Homeopathy, Naturopathy }\end{array}$ & 115 \\
\hline $\begin{array}{c}\text { Detoxification, Massage, Acupuncture, Hydrotherapy, } \\
\text { Naturopathy }\end{array}$ & 118 \\
\hline $\begin{array}{c}\text { Detoxification only } \\
\text { Homeopathy only }\end{array}$ & 22 \\
\hline Naturopathy only & 302 \\
\hline
\end{tabular}

\section{Use of single detoxification therapy}

In addition to Naturopathy, single therapy was equally used to treat patients and below is the detail:

$40 \%$ of the patients were treated with naturopathy (Figure 3) which consists of dietary measures such as "cleansing foods" increased fruit/vegetable intake, vitamin/mineral supplementation, organic foods, elimination diet, probiotics, stool bulking agents, Pyro energy, Massage, Homeopathy, Hydrotherapy, Naturopathy and acupuncture (Table 3); (Figure 4).

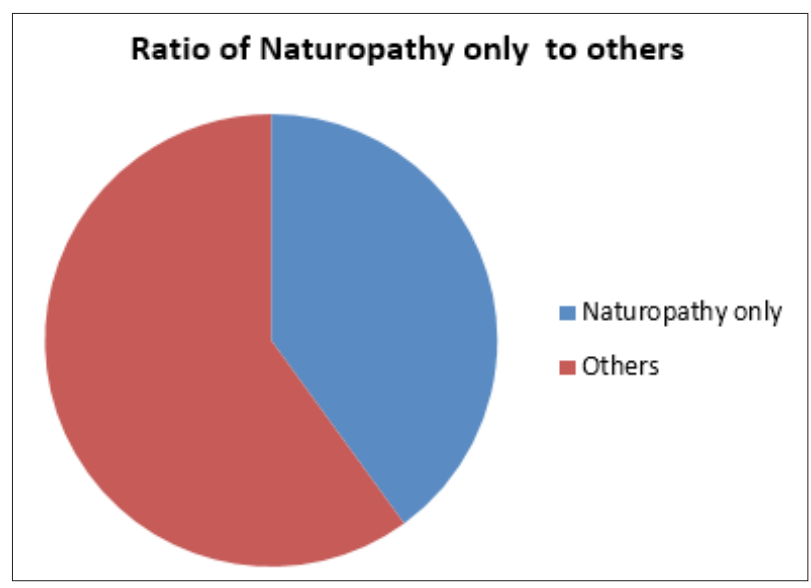

Figure 3: 
Table 3:

\begin{tabular}{|c|c|c|}
\hline Therapy & $\begin{array}{c}\text { No of Pa- } \\
\text { tients }\end{array}$ & Percentage Formation \\
\hline Homeopathy & 22 & $7 \%$ \\
\hline Naturopathy & 302 & $90 \%$ \\
\hline Detoxification & 5 & $1.50 \%$ \\
\hline Pyro energy & 1 & $0.30 \%$ \\
\hline Massage & 3 & $0.90 \%$ \\
\hline Hydrotherapy & 0 & $0 \%$ \\
\hline Acupuncture & 3 & $0.90 \%$ \\
\hline
\end{tabular}

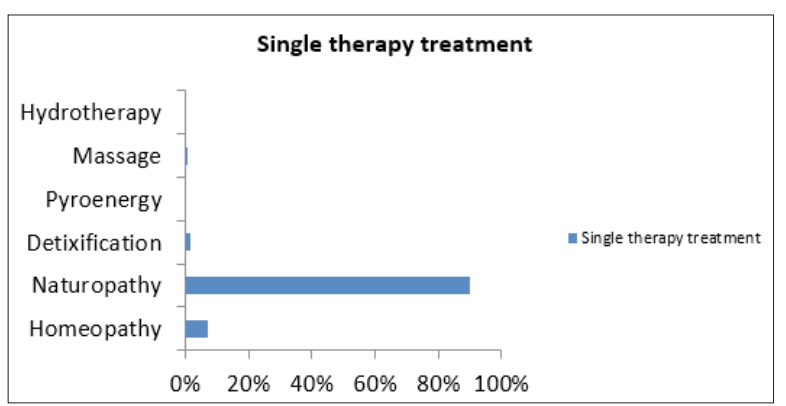

Figure 4:

\section{Diseases and diagnosis}

A number of diseases were diagnosed within this period under review. The disease diagnosed was employed to determine the need for detoxification therapies and the duration of the detoxification therapies (Table 4). On an average, about 1, 580 diseases of different levels were diagnosed using three major diagnostic techniques; medical history, environmental exposure history, and laboratory evaluations (Figure 5). The various diagnosed diseases are listed in Table 5; Figure 6 below on the bases of body systems.

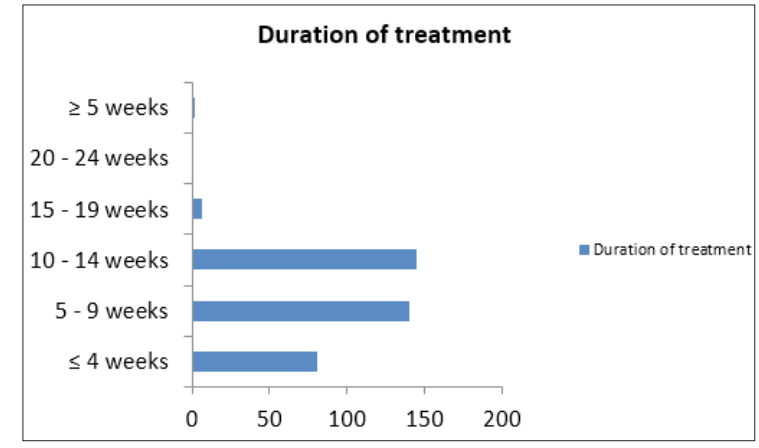

Figure 5: Duration of treatment.

Table 4: Duration of treatment.

\begin{tabular}{|c|c|c|}
\hline$\leq 4$ weeks & 81 & $9 \%$ \\
\hline $5-9$ weeks & 140 & $15 \%$ \\
\hline $10-14$ weeks & 145 & $16 \%$ \\
\hline $15-19$ weeks & 6 & $0.70 \%$ \\
\hline $20-24$ weeks & 1 & $0.10 \%$ \\
\hline$\geq 25$ weeks & 2 & $0.20 \%$ \\
\hline
\end{tabular}

\begin{tabular}{|c|c|c|}
\hline Unspecified period & 523 & $59 \%$ \\
\hline
\end{tabular}

Table 5:

\begin{tabular}{|c|c|}
\hline System/Affected Part of Body & No of Cases \\
\hline Central nervous system & 86 \\
\hline Reproductive system & 227 \\
\hline Respiratory system & 103 \\
\hline Musculoskeletal system & 338 \\
\hline Endocrine system & 37 \\
\hline Cardio vascular system & 163 \\
\hline Gastro intestinal system & 192 \\
\hline Renal & 17 \\
\hline Skin/Integument & 46 \\
\hline Others (malaria, typhoid and body pains) & 408 \\
\hline
\end{tabular}

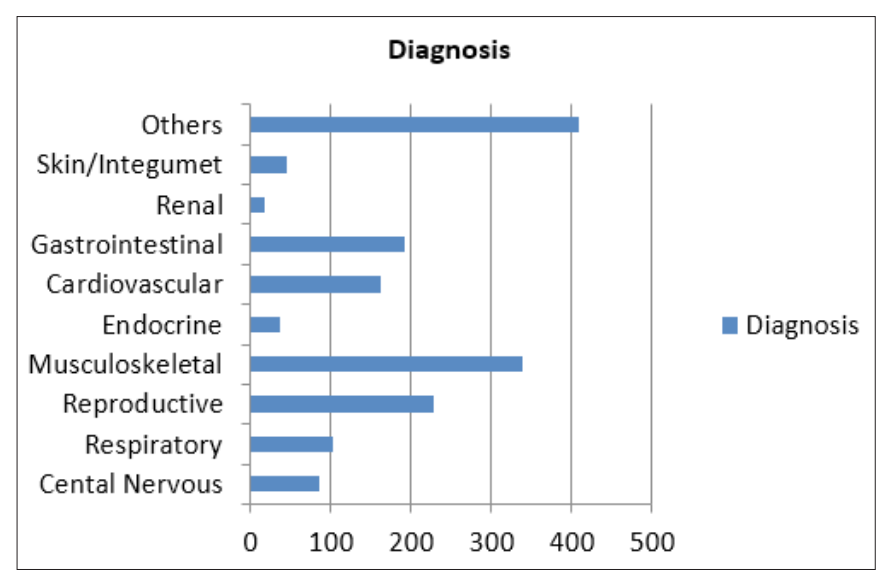

Figure 6: Summary of products of detoxification.

\section{Discussion}

Detoxification (sometimes called body cleansing) is a type of alternative medicine treatment which aims to rid the body of unspecified "toxins"-that have accumulated in the body. During a period of 10 months, 961 patients were treated with detoxification therapies at FEDCAM clinic in Abuja. Other activities commonly associated with detoxification at the clinic included dieting, consuming exclusively or avoiding specific foods (such as fats, carbohydrates, fruits, vegetables, juices, herbs, or water) and fasting. The increase in the number of patients treated when compared to the previous year's shows a remarkable increase which is a justification of the fact that a lot of people are beginning to appreciate the importance of naturopathy [7] despite the criticisms the practice has witnessed in the recent times. An aspect of the detoxification procedure as carried out at our Clinic requires the patient dipping his/her feet inside a bowl containing clean water. The water is ionized by adding a small quantity of Sodium Chloride (Table Salt). The quantity of table salt needed is at the discretion of the physician. An array is dropped inside the ionized water. The array is to serve as a medium for extracting the toxins from different organs of the body. The whole procedure lasts for 30 minutes and the color of the eventual product depends on the 
organ of the body from where the toxins are being extracted (Table 6) below enumerates this: Summary of Products of detoxification.

Table 6: Summary of products of detoxification.

\begin{tabular}{|c|c|c|c|}
\hline S/No & Colour & Organ & End Product \\
\hline 1 & Black (Deep) & Liver & Toxin \\
\hline 2 & Orange & Joints & Toxin \\
\hline 3 & Green & $\begin{array}{c}\text { Gall Blad- } \\
\text { der }\end{array}$ & Toxin \\
\hline 4 & Brown (Slight) & Liver & Toxin \\
\hline 5 & Suspended Oil Droplets & --- & Cholesterol \\
\hline 6 & Whitish Foam & $\begin{array}{c}\text { Immune } \\
\text { System }\end{array}$ & \begin{tabular}{c} 
Toxin \\
\hline 7
\end{tabular} \\
\hline \multicolumn{2}{|c|}{ Suspended Brown Particles } & -- & $\begin{array}{c}\text { Drug/Food } \\
\text { Debris }\end{array}$ \\
\hline
\end{tabular}

\section{References}

1. Wuttke W, Jarry H, Seidlova DW (2010) Definition, classification and mechanism of action of endocrine disrupting chemicals. Hormones (Athens) 9(1): 9-15.

2. Grandjean P, Bellinger D, Bergman A, Cordier S, Smith GD, et al. (2008) The faroes statement: Human health effects of developmental exposure to chemicals in our environment. Basic Clin Pharmacol Toxicol 102(2): 73-75.
3. Cummings JA, Clemens LG, Nunez AA (2010) Mother counts: how effects of environmental contaminants on maternal care could affect the offspring and future generations. Front Neuroendocrinol 31(4): 440451.

4. Soto AM, Sonnenschein C (2010) Environmental causes of cancer: endocrine disruptors as carcinogens. Nat Rev Endocrinol 6(7): 363-370.

5. Takser L, Mergler D, Baldwin M, Grosbois S, Smargiassi A, et al. (2005) Thyroid hormones in pregnancy in relation to environmental exposure to organochlorine compounds and mercury. Environ Health Perspect 113(8): 1039-1045.

6. World Health Organization (2002) Traditional Medicine Strategy 20022005.

7. Allen J, Montalto M, Lovejoy J, Weber W (2011) Detoxification in Naturopathic Medicine: A Survey. J Altern Complement Med 17(12): 1175-1180.

8. Barile FACTPaMCPLBRF (2004) Clinical toxicology principles and mechanisms. FL: CRC Press, Boca Raton, US.

9. Sullivan JB (2001) Clinical environmental health and toxic exposures. Lippincott Williams \& Wilkins, Philadelphia, US.

10. Hennig B, Ettinger AS, Jandacek RJ, Koo S, Clain C, et al. (2007) Using nutrition for intervention and prevention against environmental chemical toxicity and associated diseases. Environ Health Perspect 115(4): 493-495. 